\title{
Robust Entanglement Preparation through Spatial Indistinguishability Quantified by Entropic Measure ${ }^{\dagger}$
}

\author{
Farzam Nosrati 1,2, Alessia Castellini ${ }^{3}$, Giuseppe Compagno ${ }^{3}$ and Rosario Lo Franco 4 \\ 1 Dipartimento di Ingegneria, Università di Palermo, Viale delle Scienze, Edificio 9, Palermo, Italy \\ 2 INRS-EMT, 1650 Boulevard Lionel-Boulet, Varennes, QC, Canada \\ 3 Dipartimento di Fisica e Chimica-Emilio Segrè, Università di Palermo, via Archirafi 36, Palermo, Italy \\ 4 Dipartimento di Ingegneria, Università di Palermo, Viale delle Scienze, Edificio 6, Palermo, Italy \\ + Presented at the Entropy 2021: The Scientific Tool of the 21st Century, 5-7 May 2021; Available online: \\ https://sciforum.net/conference/Entropy2021/.
}

Published: 5 May 2021

Initialization of composite quantum systems into highly entangled states is important to enable their use for quantum technologies. However, unavoidable noise in the preparation stage makes the system state mixed, hindering the achievement of this goal. We address this problem in the context of identical particle systems adopting the operational framework of spatially localized operations and classical communication (sLOCC). After a brief description of the formalism, we define the entanglement of formation for an arbitrary state (pure or mixed) of two identical qubits, valid for both bosons and fermions. We then introduce an entropic measure of spatial indistinguishability as an information resource, tunable by the shapes of spatial wave functions. We finally apply these tools to a situation of experimental interest, that is noisy entangled state preparation. We find that spatial indistinguishability, even partial, can be a property shielding nonlocal entanglement from preparation noise, independently of the exact shape of spatial wave functions. These results prove that quantum indistinguishability is an inherent control for noise-free entanglement generation.

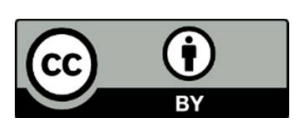

(C) 2021 by the authors. Licensee MDPI, Basel, Switzerland. This article is an open access article distributed under the terms and conditions of the Creative Commons Attribution (CC BY) license (http://creativecommons.org/licenses/by/4.0/). 